\title{
Coherence stabilization of a two-qubit gate by AC fields
}

\author{
Karen M. Fonseca Romero* Sigmund Kohler, and Peter Hänggi \\ Institut für Physik, Universität Augsburg, Universitätsstraße 1, D-86135 Augsburg, Germany
}

(Dated: June 21, 2018)

\begin{abstract}
We consider a CNOT gate operation under the influence of quantum bit-flip noise and demonstrate that ac fields can change bit-flip noise into phase noise and thereby improve coherence up to several orders of magnitude while the gate operation time remains unchanged. Within a high-frequency approximation, both purity and fidelity of the gate operation are studied analytically. The numerical treatment with a Bloch-Redfield master equation confirms the analytical results.

PACS numbers: 03.67.Pp, 05.40.-a, 42.50.Hz, 03.65.Yz
\end{abstract}

Despite the remarkable experimental realization of qubits [1, 2, 3] and two-qubit gates [4] in condensed matter systems, the construction of a working quantum computer remains an elusive goal, not only due to deficiencies of the control circuitry, but also due to the unavoidable coupling to the environment. Several proposals to overcome the ensuing decoherence have been put forward, such as the use of decoherence free subspaces [5, 6, 6, 8, 9], quantum Zeno subspaces [10], dynamical decoupling 11, 12, 13, 14], and coherent destruction of tunneling [15].

A single qubit under the influence of bit-flip noise can be modeled by the spin-boson Hamiltonian $H_{0}=$ $-\frac{\Delta}{2} \sigma^{z}+\sigma^{x} \xi$, where $\sigma^{x, z}$ denotes Pauli matrices and $\xi$ is a shorthand notation for the quantum noise specified below. The influence of the bath is governed by the spectral density of the noise at the tunneling frequency $\Delta / \hbar$. A possible driving field may couple to any projection $\vec{n}$ of the (pseudo) spin operator $\vec{\sigma}$, i.e., be proportional to $\vec{n} \cdot \vec{\sigma}$. In Ref. [15], two particular choices have been studied and compared against each other: A driving of the form $H(t)=A \sigma^{z} \cos (\Omega t)$ commutes with the static qubit Hamiltonian while it modifies the bath coupling $\sigma^{x} \xi$ in such a way that the spectral density of the bath at multiples of the driving frequency becomes relevant. For a proper driving amplitude, this eliminates noise with frequencies below the driving frequency which, thus, should lie above the cutoff frequency of the bath. This scheme represents a continuous-wave version of dynamical decoupling [15. By contrast, a driving of the type $H(t)=A \sigma^{x} \cos (\Omega t)$ renders the qubit-bath coupling unchanged but renormalizes the tunnel splitting $\Delta$ towards smaller values and thereby causes the so-called coherent destruction of tunneling (CDT) 16, 17]. Then, decoherence is determined by the spectral density of the bath at a lower effective tunnel frequency. For an ohmic bath being linear in the frequency, the consequence is that both decoherence and the coherent oscillations in the rotating frame are slowed down by the same factor 15]. Therefore, the number of coherent oscillations is not enlarged and, thus, for single-qubit operations, CDT might be of limited use.

In this work, we propose a coherence stabilization scheme for a CNOT gate based on isotropic Heisenberg interaction [18, 19]. Our scheme does not suffer from the drawbacks mentioned above because (i) it involves only intermediately large driving frequencies that can lie well below the bath cutoff and (ii) it does not increase the operation time of the gate. Since we shall employ a field that couples to the same coordinate as the quantum noise, the present coherence stabilization is different from dynamical decoupling.

CNOT gate with bit-flip noise. - We consider a pair of qubits described by the Hamiltonian [18, 19, 20, 21]

$$
H_{\text {gate }}=\frac{1}{2} \sum_{j=1,2}\left(\Delta_{j} \sigma_{j}^{x}+\epsilon_{j} \sigma_{j}^{z}\right)+J \vec{\sigma}_{1} \cdot \vec{\sigma}_{2},
$$

with a spin-spin coupling of the Heisenberg type, where $j$ labels the qubits. In order to construct a quantum gate, the tunnel splittings $\Delta_{j}$, the biases $\epsilon_{j}$, and the spin-spin coupling $J$ have to be controllable in the sense that they can be turned off and that their signs can be changed. Then, a suitable sequence of interactions yields the CNOT operation [18, 19, 20, 21.

$$
U_{\mathrm{CNOT}}^{H} \sim U_{H}(\pi / 8) U_{1 z}(\pi) U_{H}(\pi / 8),
$$

where $U_{1 z}(\varphi)=\exp \left(-i \varphi \sigma_{1}^{z} / 2\right)$ represents a rotation of qubit 1 around the $z$-axis and $U_{H}(\varphi)=\exp \left(-i \varphi \vec{\sigma}_{1} \cdot \vec{\sigma}_{2}\right)$ describes the time evolution due to the qubit-qubit interaction. The symbol $\sim$ denotes equality up to local unitary transformations, i.e., transformations which act on only one qubit. Since we focus on decoherence during the stage of the qubit-qubit interaction, we take as a working hypothesis that one-qubit operations perform ideally. Thus, decoherence takes place while $\Delta_{j}=\epsilon_{j}=0$ and $J>0$ during the required total qubit-qubit interaction time $t_{J}=\pi \hbar / 4 J$.

The bit-flip noise is specified by the system-bath Hamiltonian $H=H_{\text {gate }}+H_{\text {coupl }}+H_{\text {bath }}$ where $H_{\text {coupl }}=$ $\frac{1}{2} \sum_{j=1,2} \sigma_{j}^{x} \sum_{\nu} \hbar c_{\nu}\left(a_{j \nu}^{\dagger}+a_{j \nu}\right)$ describes the coupling of qubit $j$ to a bath of harmonic oscillators with frequencies $\omega_{\nu}$ and $H_{\text {bath }}=\sum_{j \nu} \hbar \omega_{\nu} a_{j \nu}^{\dagger} a_{j \nu}$. The bath is fully specified by its spectral density $I(\omega)=\pi \sum_{\nu} c_{\nu}^{2} \delta\left(\omega-\omega_{\nu}\right)$. Within the present work, we consider the so-called ohmic 
spectral density $I(\omega)=2 \pi \alpha \omega \exp \left(-\omega / \omega_{c}\right)$ with the dimensionless coupling strength $\alpha$ and the cutoff frequency $\omega_{c}$. In order to complete the model, we specify the initial condition for the total density matrix to be of the Feynman-Vernon type, i.e., initially, the bath is in thermal equilibrium and uncorrelated with the system, $\rho_{\text {tot }}\left(t_{0}\right)=\rho\left(t_{0}\right) \otimes R_{\text {bath,eq }}$, where $\rho$ is the reduced density operator of the two qubits and $R_{\text {bath,eq }} \propto$ $\exp \left(-H_{\text {bath }} / k_{B} T\right)$ is the canonical ensemble of the bath.

If $\alpha k_{B} T$ is larger than the typical system energy $J$ and the dissipation strength is sufficiently small, $\alpha \ll 1$, the dissipative system dynamics is well described within a Born-Markov approach. There, one starts from the Liouville-von Neumann equation $i \hbar \dot{\rho}_{\text {tot }}=\left[H, \rho_{\text {tot }}\right]$ for the total density operator and obtains by standard techniques the master equation 22]

$$
\begin{aligned}
\dot{\rho} & =\frac{1}{i \hbar}\left[H_{\text {gate }}, \rho\right]-\sum_{j}\left[\sigma_{j}^{x},\left[Q_{j}(t), \rho\right]\right]-\sum_{j}\left[\sigma_{j}^{x},\left\{P_{j}(t), \rho\right\}\right] \\
& \equiv \frac{1}{i \hbar}\left[H_{\text {gate }}, \rho\right]-\Lambda(t) \rho
\end{aligned}
$$

with the anti-commutator $\{A, B\}=A B+B A$ and

$$
Q_{j}(t)=\frac{1}{4 \pi} \int_{0}^{\infty} d \tau \int_{0}^{\infty} d \omega \mathcal{S}(\omega) \cos (\omega \tau) \widetilde{\sigma}_{j}^{x}(t-\tau, t) .
$$

Here, $\mathcal{S}(\omega)=I(\omega) \operatorname{coth}\left(\hbar \omega / 2 k_{B} T\right)$ is the Fourier transformed of the symmetrically-ordered equilibrium bath correlation function $\frac{1}{2}\langle\{\xi(\tau), \xi(0)\}\rangle_{\text {eq }}$ of the collective coordinate $\xi=\sum_{\nu} c_{\nu}\left(a_{\nu}^{\dagger}+a_{\nu}\right)$. The notation $\widetilde{X}\left(t, t^{\prime}\right)$ is a shorthand for the Heisenberg operator $U^{\dagger}\left(t, t^{\prime}\right) X U\left(t, t^{\prime}\right)$ with $U$ being the propagator of the coherent system dynamics. Note that $\mathcal{S}(\omega)$ and $I(\omega)$ are the same for both qubits due to the assumption of two identical environments. Replacing in Eq. (4) the term $\mathcal{S}(\omega) \cos (\omega \tau)$ by $I(\omega) \sin (\omega \tau)$, yields the operator $P_{j}(t)$. We emphasize that the particular form (3) of the master equation is valid also for an explicitly time-dependent Hamiltonian.

The heat baths, whose influence is described by the second and third term of the master equation (3), lead to decoherence, i.e., the evolution from a pure state to an incoherent mixture. Decoherence can be measured by the decay of the purity $\operatorname{tr}\left(\rho^{2}\right)$ from the ideal value 1 . The gate purity (later refered to as "purity") $\mathcal{P}(t)=\overline{\operatorname{tr}\left(\rho^{2}(t)\right)}$, which characterizes the gate independently of the specific input, results from the ensemble average over all pure initial states [23]. For weak dissipation, the purity is determined by its time derivative at initial time. Performing the ensemble average, one finds the gate purity decay

$$
\left.\dot{\mathcal{P}}(t)\right|_{t=0}=-2 \overline{\operatorname{tr}(\rho \Lambda \rho)}=-\frac{2}{d(d+1)} \operatorname{Tr} \Lambda,
$$

where $d=4$ is the dimension of the system Hilbert space and $\operatorname{Tr}$ denotes the trace in superoperator space defined as $\operatorname{Tr} \Lambda=\sum_{a, a^{\prime}} \operatorname{tr}\left(\left|a^{\prime}\right\rangle\langle a|\Lambda| a\rangle\left\langle a^{\prime}\right|\right)$ with $\{|a\rangle\}$ being an arbitrary orthonormal basis of the system Hilbert space. It can be shown that the term containing $P_{j}$ does not contribute to $\operatorname{Tr} \Lambda$.

In order to evaluate for our setup the purity decay, we need explicit expressions for the operators $Q_{j}$ and, thus, have to compute the Heisenberg operators $\widetilde{\sigma}(t-\tau, t)$ for the Hamiltonian $H_{0}=J \vec{\sigma}_{1} \cdot \vec{\sigma}_{2}$. This calculation is most conveniently done in the eigenbasis of $H_{0}$, i.e. in the basis of the total (pseudo) spin $\vec{L}=\frac{1}{2}\left(\vec{\sigma}_{1}+\vec{\sigma}_{2}\right)$. After performing the time and the frequency integration in Eq. (4), we finally arrive at $\dot{\mathcal{P}}=-\frac{2}{5}\{\mathcal{S}(0)+\mathcal{S}(4 J / \hbar)\}$, where we have ignored Lamb shifts and defined $\mathcal{S}(0) \equiv \lim _{\omega \rightarrow 0} \mathcal{S}(\omega)=$ $4 \pi \alpha k_{B} T / \hbar$. In particular, we find that for low temperatures, $k_{B} T \lesssim J$, decoherence is dominated by $\mathcal{S}(4 J / \hbar)$ such that $\dot{\mathcal{P}} \approx-16 \pi \alpha J / 5 \hbar$. This value reflects the influence of the so-called quantum noise which cannot be reduced by further cooling.

$A C$ driving field. - In order to manipulate the coherence properties, we act upon qubit 1 by an ac field which causes a time-dependent level splitting according to

$$
H_{\mathrm{ac}}(t)=f(t) \sigma_{1}^{x},
$$

where $f(t)$ is a $T$-periodic function with zero timeaverage. Due to the finite driving time, which acts only while the Heisenberg coupling is switched on, the spectrum of the driving field acquires a dispersion $\Delta \omega \approx \Omega / k$, where $k$ is the number of driving periods. To keep the influence of the dispersion small, we have to choose $k \gg 1$.

Next, we derive within a high-frequency approximation analytical expressions for both the coherent propagator $U\left(t, t^{\prime}\right)$ and the purity decay (5). We start out by transforming the total Hamiltonian into a rotating frame with respect to the driving by the unitary transformation

$$
U_{\mathrm{ac}}(t)=e^{-i \phi(t) \sigma_{1}^{x}}, \quad \phi(t)=\frac{1}{\hbar} \int_{0}^{t} d t^{\prime} f\left(t^{\prime}\right),
$$

which yields the likewise $T$-periodic Hamiltonian $\widetilde{H}(t)=$ $U_{\mathrm{ac}}^{\dagger}(t) H_{\text {gate }} U_{\text {ac }}(t)$. For large driving frequencies $\Omega \gg J$, it is possible to separate time scales and thereby replace $\widetilde{H}(t)$ by its time-average

$$
\bar{H}=\left(J-J_{\perp}\right) \sigma_{1}^{x} \sigma_{2}^{x}+J_{\perp} \vec{\sigma}_{1} \cdot \vec{\sigma}_{2},
$$

where the constant $J_{\perp}=J\langle\cos [2 \phi(t)]\rangle_{T}$ denotes an effective interaction "transverse" to the driving and $\langle\ldots\rangle_{T}$ the time average over the driving period. Within this approximation, the propagator of the driven system reads

$$
U_{\mathrm{eff}}\left(t, t^{\prime}\right)=e^{-i \phi(t) \sigma_{1}^{x}} e^{-i \bar{H}\left(t-t^{\prime}\right) / \hbar} e^{i \phi\left(t^{\prime}\right) \sigma_{1}^{x}} .
$$

Having this propagator at hand, we are in the position to derive explicit expressions for the operators $\sigma_{j}^{x}(t-\tau, t)$ and $Q_{j}$ and, consequently, also for the generator of the dissipative dynamics $\Lambda$. The calculation is conveniently done in the basis of the total spin $\vec{L}$ and $L_{x}$ which, owing 
to the relation $\sigma_{1}^{x} \sigma_{2}^{x}=\frac{1}{2}\left(\sigma_{1}^{x}+\sigma_{2}^{x}\right)^{2}-1$, is an eigenbasis of the Hamiltonian (8). We insert the resulting expression for $\Lambda$ into Eq. (5) and finally obtain the purity decay

$$
\dot{\mathcal{P}}=-\frac{2}{5}\left\{\mathcal{S}(0)+\mathcal{S}\left(4 J_{\perp} / \hbar\right)\right\} .
$$

For $f(t) \equiv 0$, we find $J_{\perp}=J$ such that Eq. (10) agrees with what we found in the static case; otherwise, the inequality $\left|J_{\perp}\right|<J$ holds and, thus, the bath correlation function $\mathcal{S}$ in Eq. (10) has to be evaluated at a lower frequency. For an ohmic or a super-ohmic bath, $\mathcal{S}(\omega)$ is a monotonously increasing function and, consequently, the ac field reduces $\dot{\mathcal{P}}$ (unless $J>\omega_{\text {cutoff }}$ ).

The purity decay assumes its minimum for $J_{\perp}=0$. This condition marks the working points on which we shall focus in the following. For an ohmic spectral density $I(\omega)=2 \pi \alpha \omega$, the purity decay at the working points becomes $\dot{\mathcal{P}}=-\frac{4}{5} \mathcal{S}(0)=-8 \pi \alpha k_{B} T / 5$ for all temperatures. This particular value has to be compared to the purity decay in the absence of driving: An analysis reveals that for $k_{B} T>J$, decoherence is essentially driving independent. By contrast for low temperatures, $k_{B} T<J$, the driving reduces the decoherence rate by a factor $k_{B} T / 2 J$. The reason for this low-temperature behavior is that for $J_{\perp}=0$, the coupling operators $\sigma_{j}^{x}$ commute with the effective Hamiltonian (8) such that noise acts as pure phase noise with a strength proportional to the temperature.

For a rectangular driving for which $f(t)$ switches between the values $\pm A / 2$, the condition $J_{\perp}=0$ yields $A=\hbar \Omega$ which corresponds to the application of two $\pi$-pulses per period. For a harmonic driving, $f(t)=$ $A \cos (\Omega t) / 2$, one obtains $J_{\perp}=J J_{0}(A / \hbar \Omega)$, where $J_{0}$ denotes the zeroth-order Bessel function of the first kind. Then, at the working points $J_{\perp}=0$, the ratio $A / \hbar \Omega$ assumes a zero of $J_{0}$, i.e., one of the values $2.405 . ., 5.520$.., $8.654 \ldots, \ldots$...

So far, we focussed on decoherence and ignored the influence of the driving on the coherent dynamics. Since the driving changes also the coherent dynamics, the pulse sequence of the CNOT operation needs a modification: At the working points of the driven system, the propagator (9) becomes $U_{\text {eff }}\left(t, t^{\prime}\right)=\exp \left[-i J \sigma_{1}^{x} \sigma_{2}^{x}\left(t-t^{\prime}\right) / \hbar\right]$ and, thus, represents the time evolution caused by a socalled Ising interaction $J \sigma_{1}^{x} \sigma_{2}^{x}$. This time evolution allows one to implement the alternative CNOT operation $U_{\text {CNOT }}^{I} \sim \exp \left(-i \pi \sigma_{1}^{x} \sigma_{2}^{x} / 4\right)=U_{\text {eff }}\left(t+t_{J}, t\right)$ [21, 24]. Note that the interaction time $t_{J}=\pi \hbar / 4 J$ is the same as for the original gate operation $U_{\mathrm{CNOT}}^{H}$. Since $U_{\mathrm{ac}}(2 \pi / \Omega)$ is the identity [cf. Eq. (7)], we assume for convenience that the driving period $2 \pi / \Omega$ is an integer multiple of $t_{J}$, i.e. $\Omega=8 k J / \hbar$ with integer $k$.

Numerical solution. - The calculation of the purity decay presented above relies on a high-frequency approximation which is correct only to lowest order in $J / \hbar \Omega$. Thus, these should be compared to the exact numerical solution of the master equation (3). An ef-

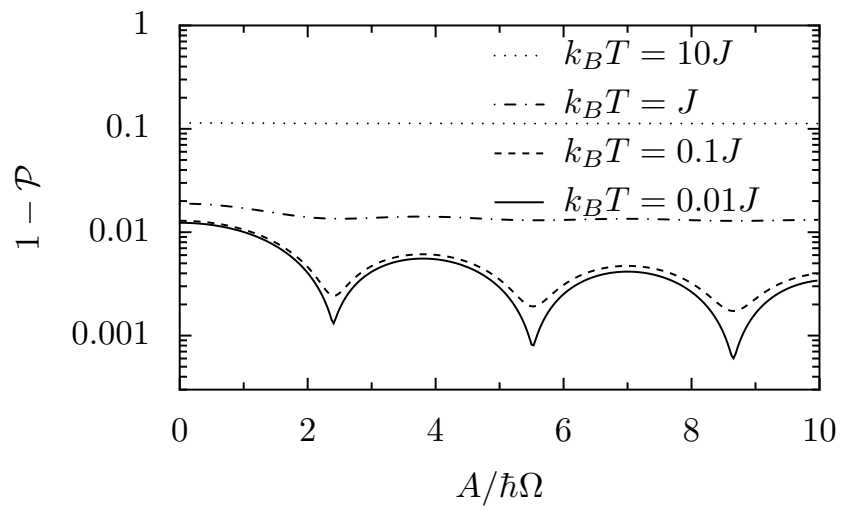

FIG. 1: Purity loss $1-\mathcal{P}$ during the Heisenberg interaction time $t_{J}$ as a function of the driving amplitude for various temperatures. The driving frequency is $\Omega=32 \mathrm{~J} / \hbar$ and the dissipation strength $2 \pi \alpha=0.01$. For $A=0$, the undriven situation is reproduced.

ficient scheme for that purpose is a modified BlochRedfield formalism whose cornerstone is a decomposition into the Floquet basis of the driven system [22]: According to the Floquet theorem, the Schrödinger equation of a periodically driven quantum system possesses a complete set of solutions of the form $\left|\psi_{\alpha}(t)\right\rangle=$ $\exp \left(-i \epsilon_{\alpha} t / \hbar\right)\left|\phi_{\alpha}(t)\right\rangle$, where the so-called Floquet states $\left|\phi_{\alpha}(t)\right\rangle$ obey the time-periodicity of the Hamiltonian and $\epsilon_{\alpha}$ denotes the so-called quasienergy. They are elements of an Hilbert space extended by a $T$-periodic time coordinate and are computed from the eigenvalue equation $[H(t)-i d / d t]\left|\phi_{\alpha}(t)\right\rangle=\epsilon_{\alpha}\left|\phi_{\alpha}(t)\right\rangle$. In the Floquet basis $\left\{\left|\phi_{\alpha}(t)\right\rangle\right\}$, the master equation (3) obtains the form $\dot{\rho}_{\alpha \beta}=-\frac{i}{\hbar}\left(\epsilon_{\alpha}-\epsilon_{\beta}\right) \rho_{\alpha \beta}-\sum_{\alpha^{\prime} \beta^{\prime}} \Lambda_{\alpha \beta, \alpha^{\prime} \beta^{\prime}} \rho_{\alpha^{\prime} \beta^{\prime}}$. The benefit of this representation is that the time dependence of the original Hamiltonian has been eliminated by choosing a suitable basis. Moreover, for weak dissipation, we can replace within a rotating-wave approximation $\Lambda(t)$ by its time average 22. Finally, we integrate the master equation to obtain the dissipative propagator which in turn allows to evaluate all quantities of interest.

In our numerical studies, we restrict ourselves to purely harmonic driving $f(t)=A \cos (\Omega t) / 2$. The resulting purity loss during the interaction time $t_{J}$ is depicted in Fig. 1] We find that for $k_{B} T>J$, decoherence is fairly independent of the driving. This behavior changes as the temperature is lowered: Once $k_{B} T<J$, the purity loss is significantly reduced whenever the ratio $A / \hbar \Omega$ is close to a zero of the Bessel function $J_{0}$. Both observations confirm the preceding analytical estimates. The behavior at the first working point $A \approx 2.4 \hbar \Omega$ is depicted in Fig. 2(a). For relatively low driving frequencies, we find the purity loss being proportional to $J / \Omega$. There are significant deviations from the analytical result for small $\Omega$ because the low-frequency regime is not within the scope of our analytical treatment. With increasing 


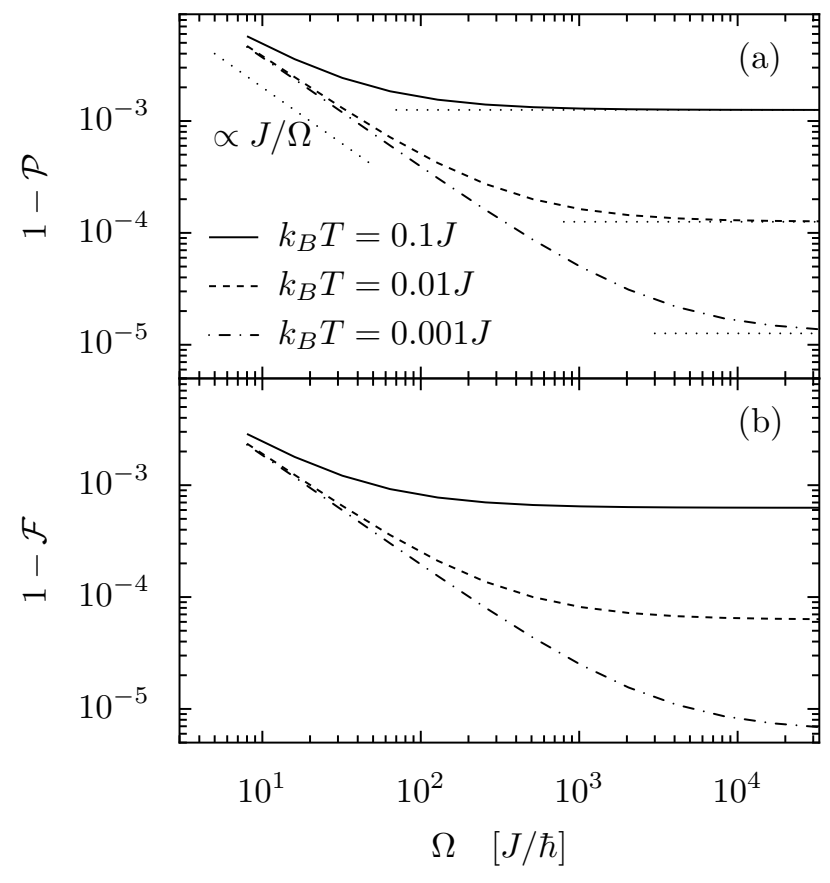

FIG. 2: (a) Purity loss during the Heisenberg interaction as a function of the driving frequency. The driving amplitude is adjusted such that $1-\mathcal{P}$ assumes its first minimum; i.e. $A \approx 2.4 \hbar \Omega$, cf. Fig. 11 The dotted lines mark the analytical estimate $1-\mathcal{P}\left(t_{J}\right) \approx-\dot{\mathcal{P}}(0) t_{J}$ and the proportionality to $J / \Omega$. (b) Corresponding fidelity defect $1-\mathcal{F}$.

driving frequency, the discrepancy decreases until finally thermal noise dominates decoherence. The numerical solution confirms almost perfectly the results from the highfrequency approximation.

Still, there remains one caveat: The gate operation $U_{\text {CNOT }}^{I}$ relies on the fact that $U_{\text {eff }}$ is a good approximation for the dynamics of the driven gate because any deviation represents a coherent error. Therefore, we still have to demonstrate that such coherent errors are sufficiently small. As a measure for this, we employ the so-called fidelity $\mathcal{F}=\overline{\operatorname{tr}\left[\rho_{\text {ideal }} \rho\left(t_{J}\right)\right]}$ 23] which constitutes the overlap between the real outcome of the operation, $\rho\left(t_{J}\right)$, and the desired final state $\rho_{\text {ideal }}=U_{I}(\pi / 4) \rho_{\text {in }} U_{I}^{\dagger}(\pi / 4)$ in the average over all pure initial states. An ideal operation is characterized by $\mathcal{F}=1$. Here, $U_{I}(\varphi)=\exp \left(-i \varphi \sigma_{1}^{x} \sigma_{2}^{x}\right)$ denotes the propagator due to an ideal Ising spin-spin interaction. Figure 2(b) shows the fidelity defect $1-\mathcal{F}$ of the real gate operation at the first working point. In the relevant temperature regime $k_{B} T<J$, we find the crucial result that the fidelity defect is of the same order or even smaller than the purity loss. Thus, we can conclude that coherent errors are not of a hindrance.

In summary, we have put forward a strategy for reducing decoherence in a two-qubit quantum gate during the stage of qubit-qubit interaction. This coherence stabilization scheme is different from previous proposals in two respects: First, it does not rely on the manipulation of the coordinate that couples the qubits to the environment. By contrast, the central idea of our scheme is rather to suppress the coherent system dynamics "transverse" to this sensitive system coordinate. In that way, the bit-flip noise, which produces unwanted transitions, is turned into pure phase noise which is proportional to the temperature. This enables a coherence gain by cooling. The second difference is that the proposed scheme eliminates also the noise coming from the spectral range above the driving frequency and, thus, is particularly suited for ohmic noise spectra with large cutoff frequencies. Moreover, the driven system still allows one to perform the desired CNOT operation with high fidelity and within the same operation time as in the absence of the control field. Hence, the gained coherence time fully contributes to the number of feasible gate operations.

This work has been supported by the Freistaat Bayern via the quantum information initiative "Quanteninformation längs der A8" and by the Deutsche Forschungsgemeinschaft through SFB 631.

* On leave from Departamento de Física, Universidad Nacional, Bogotá, Colombia.

[1] Y. Nakamura et al., Nature 398, 786 (1999).

[2] D. Vion et al., Science 296, 886 (2002).

[3] I. Chiorescu et al., Science 299, 1869 (2003).

[4] T. Yamamoto et al., Nature 425, 941 (2003).

[5] G. M. Palma et al., Proc. R. Soc. London Ser. A 452, 567 (1996).

[6] L.-M. Duan and G.-C. Guo, Phys. Rev. Lett. 79, 1953 (1997).

[7] P. Zanardi and M. Rasetti, Phys. Rev. Lett. 79, 3306 (1997).

[8] D. A. Lidar et al., Phys. Rev. Lett. 81, 2594 (1998).

[9] A. Beige et al., Phys. Rev. Lett. 85, 1762 (2000).

[10] P. Facchi and S. Pascazio, Phys. Rev. Lett. 89, 080401 (2002).

[11] L. Viola et al., Phys. Rev. Lett. 82, 2417 (1999).

[12] D. Vitali and P. Tombesi, Phys. Rev. A 65, 012305 (2002).

[13] H. Gutmann et al., cond-mat/0308107

[14] G. Falci et al., cond-mat/0312442

[15] K. M. Fonseca Romero et al., Chem. Phys. 296, 307 (2004).

[16] F. Grossmann et al., Phys. Rev. Lett. 67, 516 (1991).

[17] M. Grifoni and P. Hänggi, Phys. Rep. 304, 229 (1998).

[18] D. Loss and D. P. DiVincenzo, Phys. Rev. A 57, 120 (1998).

[19] B. Kane, Nature (London) 393, 133 (1998).

[20] M. Thorwart and P. Hänggi, Phys. Rev. A 65, 012309 (2002).

[21] Y. Makhlin et al., Rev. Mod. Phys. 73, 357 (2001).

[22] S. Kohler et al., Phys. Rev. E 55, 300 (1997).

[23] J. F. Poyatos et al., Phys. Rev. Lett. 78, 390 (1997).

[24] N. Gershenfeld and I. L. Chuang, Science 275, 350 (1997). 